Boise State University

ScholarWorks

Geosciences Faculty Publications and

Presentations

$5-2008$

Application of Time-Lapse ERT Imaging to Watershed

Characterization

Carlyle R. Miller

Boise State University

Partha S. Routh

Boise State University

Troy R. Brosten

Boise State University

James P. McNamara

Boise State University 


\title{
Application of time-lapse ERT imaging to watershed characterization
}

\author{
Carlyle R. Miller ${ }^{1}$, Partha S. Routh ${ }^{2}$, Troy R. Brosten ${ }^{3}$, and James P. McNamara ${ }^{3}$
}

\begin{abstract}
Time-lapse electrical resistivity tomography (ERT) has many practical applications to the study of subsurface properties and processes. When inverting time-lapse ERT data, it is useful to proceed beyond straightforward inversion of data differences and take advantage of the time-lapse nature of the data. We assess various approaches for inverting and interpreting time-lapse ERT data and determine that two approaches work well. The first approach is model subtraction after separate inversion of the data from two time periods, and the second approach is to use the inverted model from a base data set as the reference model or prior information for subsequent time periods. We prefer this second approach. Data inversion methodology should be considered
\end{abstract}

when designing data acquisition; i.e., to utilize the second approach, it is important to collect one or more data sets for which the bulk of the subsurface is in a background or relatively unperturbed state. A third and commonly used approach to time-lapse inversion, inverting the difference between two data sets, localizes the regions of the model in which change has occurred; however, varying noise levels between the two data sets can be problematic. To further assess the various time-lapse inversion approaches, we acquired field data from a catchment within the Dry Creek Experimental Watershed near Boise, Idaho, U.S.A. We combined the complimentary information from individual static ERT inversions, time-lapse ERT images, and available hydrologic data in a robust interpretation scheme to aid in quantifying seasonal variations in subsurface moisture content.

\section{INTRODUCTION}

Advances in geophysical instrumentation in the past decade have made it possible to efficiently acquire large data sets in a fraction of the time compared to collecting the same set of measurements using older equipment. Modernized instrumentation also means that, in many cases, repeatability of measurements has improved, making time-lapse geophysical surveys much more practical by improving the signal-to-noise ratio.

The advantage of time-lapse measurements versus a single, static survey is that they provide a means of imaging not only subsurface properties, but also dynamic changes in these properties, which can in turn provide insight into ongoing subsurface processes. Timelapse geophysical measurements have been shown to be successful in monitoring and understanding physical processes in the subsurface, e.g. (Ramirez et al., 1993, 1995; Lumley, 2001; Tsourlos et al.,
2003; Singha and Gorelick, 2005; Lane et al., 2006; MacBeth et al., 2006; Anno and Routh, 2007).

In a general sense, time-lapse methodologies can be utilized to determine the rate at which a process is occurring, define the volume of subsurface region affected by a particular process, and understand the complex interactions between various subsurface processes. Time-lapse is especially important for near-surface studies since the medium is much more dynamic due to the proximity of the air-earth interface. This is evidenced by increase in time-lapse applications for near-surface geophysical problems (Day-Lewis et al., 2002, 2003; Singha and Gorelick, 2005).

Time-lapse geophysical measurements can help to enhance our understanding of a particular site but can also make designing our temporal and spatial sampling schemes more challenging. Ultimately, the rate at which a process of interest is occurring determines how closely spaced our data collection must occur temporally. Day-

Manuscript received by the Editor 18 July 2007; revised manuscript received 5 December 2007; published online 8 May 2008.

${ }^{1}$ Formerly Boise State University, Department of Geosciences, Boise, Idaho, U.S.A.; presently MSE Technology Applications Inc., Earth Sciences, Butte, Montana, U.S.A. E-mail: carl.miller@mse-ta.com.

${ }^{2}$ Formerly Boise State University, Department of Geosciences, Boise, Idaho, U.S.A.; presently Subsurface Technology, ConocoPhillips, Houston, Texas, U.S.A.E-mail: partha.s.routh@conocophillips.com.

${ }^{3}$ Boise State University, Department of Geosciences, Boise, Idaho, U.S.A.E-mail: troybrosten@ mail.boisestate.edu; jmcnamar@boisestate.edu. (C) 2008 Society of Exploration Geophysicists. All rights reserved. 
Lewis et al. $(2002,2003)$ demonstrate the importance of accounting for the finite time required for data collection (the data may be changing faster than we are able to complete a subset of measurements) when inverting time-lapse crosswell radar data from a tracer test.

On the other end of the temporal spectrum are long-term studies where the properties of interest vary over diurnal, seasonal, or even longer time scales. In this type of investigation, there is adequate time to collect the data with little concern as to short-timescale variations in the subsurface. The methodologies and data examples presented herein focus on studies pertaining to the latter types of processes.

Electrical resistance tomography (ERT) data are useful in imaging properties and processes associated with groundwater and unsaturated zone systems (Slater et al., 1997; Zhou et al., 2001; Binley et al., 2002; Müller et al., 2003; Mohnke et al., 2006; Oldenborger et al., 2007a, b; Descloitres et al., 2007). The ERT data are sensitive to the subsurface geoelectrical structure, which is in turn sensitive to subsurface variations in water saturation and pore water salinity (Archie, 1942; Mualem and Friedman, 1991; Henry, 1997; Ewing and Hunt, 2006). This makes ERT particularly useful for characterizing infiltration into bedrock where conventional methods of soil moisture accounting fail.

Geophysical methods have been widely used in groundwater investigations (Fitterman and Stewart, 1986; McNeill, 1990; Hubbard et al., 2001) and can provide information over large areas at a relatively inexpensive cost compared to other methods (e.g. borehole drilling and trenching). Surface ERT is minimally invasive and thus does not disturb ongoing hydrological processes at the site. Previous studies have successfully used electromagnetic and electrical methods within aquifer regions composed of fractured media to provide valuable information for hydrogeological and environmental studies (Lane et al., 1995; Hautot et al., 2002; Sharma and Baranwal, 2005; Boadu et al., 2005; Porsani et al., 2005; Hubbard and Rubin, 2006).

Bedrock infiltration is a key component of the water balance of mountain watersheds. However, it is a challenging process to measure at watershed scales and is typically calculated as a water balance residual. The primary difficulty arises from the non-Darcian flow in fractures masked by a soil mantle. Time-lapse ERT can be used to characterize the bedrock fracture networks by monitoring the changes in water saturation.

In the field data example presented in this paper, the fieldwork was timed such that we were able to collect a base data set prior to onset of winter rain and snowfall. For these background data, we assume that the in situ water saturation was at or near its annual low value. We then collected two data sets during the wet winter/spring time period to capture the changes in the electrical structure induced by the increase in water saturation. The final data set was collected late in the summer when the hydrologic system had returned to a dry state.

When collecting time-lapse geophysical data, it is important to have knowledge of the current state of the study site so that proper interpretation of the results is possible. When possible, data collection should be timed to capture at least one entire cycle of the hydrological process of interest. Data acquired over two or more entire cycles may prove useful in assessing longer-term stability of the hydrological process. With this view we acquired another survey in September 2007 to validate the hydrologic cycle stability. In addition to the ERT data collected specifically for this study, we also present soil moisture content data collected concurrently for other studies that proved useful when interpreting the results. data:

We present three approaches for inverting time-lapse geophysical

1) Model subtraction after separate inversion of the data from two time periods

2) Use of an inverted model from a base data set as the reference model for subsequent time periods

3) Inversion of the differences between two data sets

Using a combination of synthetic and field ERT data, we demonstrate that the first two approaches work well in most instances. The second approach is shown to be the preferred method. The third approach localizes the regions of the model in which change has occurred but varying noise levels between the two data sets can be problematic because of repeatability issues.

The field data example comes from a small, semiarid catchment in the Dry Creek Experimental Watershed (DCEW) near Boise, Idaho U.S.A. This study area presented an opportunity to compare and interpret geophysical results in conjunction with long-term hydrological and geological data collected at the site. We focus primarily on soil moisture content data from the site; but local precipitation, hydrogeochemical, hydraulic, and soil composition estimates also aided in the ERT data interpretations. Combining the information from these previous/ongoing studies with the individual ERT inversions and the time-lapse images, we were able to determine possible fracture locations and identify pathways of bedrock infiltration at the study site.

\section{METHODS}

\section{Electrical resistivity tomography: Acquisition and processing}

ERT measurements are highly sensitive to saturated pore spaces. With the exception of conductive rocks such as ore bodies, most rock types in the near surface under dry conditions are typically resistive, therefore propagation of electrical current in the shallow subsurface is primarily achieved via movement of ions within pore water. Assuming that the pore water preferentially flows through the bedrock fracture networks, electrical potentials will be sensitive to and help locate fracture zones within the bedrock.

The instrument used for ERT data acquisition was the IRIS Syscal Pro Switch 72. This instrument has been designed for high productivity resistivity and IP measurements with a precision of $0.2 \%$ and threshold voltage of $1 \mu \mathrm{V}$. The system features an internal switching board for 72 electrodes and an internal $250 \mathrm{~W}$ power source. The system is designed to make resistivity measurements at selected injection electrodes and potential measuring electrodes predefined by the user. Input specifications include electrode array type, combinations of electrode spacing, injection current strength, and the number of measurements to be stacked.

When data acquisition begins, the instrument checks the electrodes for contact and then takes measurements according to the user input acquisition sequence. For example, in a dipole-dipole survey such as was employed in this paper, the sequence might specify the first two electrodes as the first current injection pair. Voltages are then recorded for the remaining electrode pairs for a number of predefined combinations. Because the instrument has 10 recording channels, it is efficient to collect 10 potential measurements for each 
current injection pair. The next two electrodes inline then become the current injection pair and the process is repeated until the injection pair reaches the far end of the survey line.

Typically, ERT data quality is improved by stacking several measurements for each quadripole (transmitter-receiver pair). A data repeatability threshold (e.g., 3\%-5\% standard deviation) can then be used to remove noisy measurements from the data. Alternatively, the noisier data may be retained and assigned higher standard deviations prior to inversion. If reciprocal measurements are collected, these can also be used to eliminate noisy data or for error assignment. The electrical potentials, input current, and electrode geometry are then used to compute apparent resistivities for input to an inversion algorithm.

\section{Inversion of ERT data}

Construction of subsurface images from ERT data is a nonlinear inverse problem with the goal of recovering the model (electrical conductivities) that reproduces the observed data (electrical potentials). The observed data are assumed to be contaminated with noise; therefore an exact fit of the model to the data would result in extraneous structure. Typically, some level of noise is assumed for each datum, and then a model objective function that penalizes the smallness and flatness of the model is minimized to reproduce data only to within an amount that is justified by the amount of noise in the data. The amount of structure in the final model is determined by how well the observed data are reproduced (Oldenburg and Li, 1994).

An important consideration of any geophysical imaging application is to determine which features within the model are required to fit the data (Miller and Routh, 2007). To determine this, and subsequently the depth of investigation, one can invert the same data set using two different reference (background) models. The model regions that are not constrained by the data will revert back to the reference model (Oldenburg and Li, 1999).

For all of the examples in this paper, inverse modeling was carried out using DCIP2D software (developed by the University of British Columbia), which uses a 2D finite volume method to model the DC potentials. The inversion recovers $\ln (\sigma)$, allowing for a large range of conductivities as well as imposing positivity. The inverse problem can be stated as

$$
\begin{gathered}
\operatorname{minimize} \psi_{m}=\alpha_{s}\left\|W_{s}\left(m-m_{0}\right)\right\|^{2}+\alpha_{x}\left\|W_{x}\left(m-m_{0}\right)\right\|^{2} \\
+\alpha_{z}\left\|W_{z}\left(m-m_{0}\right)\right\|^{2} \\
\text { subject to } \psi_{d}=\left\|W_{d}\left(d^{\text {pred }}-d^{\text {obs }}\right)\right\|^{2}=\psi_{d}^{*}
\end{gathered}
$$

This inverse problem is solved by minimizing,

$$
\psi\left(m, m_{0}\right)=\beta\left\|W_{m}\left(m-m_{0}\right)\right\|^{2}+\left\|W_{d}\left(d^{\text {pred }}-d^{\text {obs }}\right)\right\|^{2} .
$$

In the preceding equations, $m$ is the model sought, $m_{0}$ is the reference model, $d_{\text {obs }}$ is the observed data, $d_{\text {pred }}$ is the predicted data, and $\psi_{d}^{*}$ is the tolerance for the data misfit, chosen, e.g., based on a $\chi^{2}$ misfit criterion. The three terms in the model objective function $\left(\psi_{m}\right.$, equation 1) allow us to apply smallness and smoothness constraints to the solution, while the data objective function $\left(\psi_{d}\right.$, equation 2$)$ ensures that we honor the data observations while accounting for noise in the data, and $\beta$ (from equation 3 ) is the regularization parameter that defines the trade-off between fitting the data and honoring the a priori constraints.

The data weighting matrix $W_{d}$ is diagonal and we chose to use the reciprocals of the data standard deviations as the weights. $W_{s}$ is diagonal, and $W_{x}$ and $W_{z}$ are finite-difference operators. The $\alpha$ parameters are used to control the relative contribution of smallest and flattest model constraints. Note that $\beta\left\|W_{m}\left(m-m_{0}\right)\right\|^{2}$ in equation 3 is a shorthand notation for the right-hand side of equation 1. For additional details regarding the inversion, see Oldenburg and $\mathrm{Li}$ (1994).

\section{Noise considerations}

Some of the major sources of noise in a DC resistivity experiment are high contact resistance, measurement errors, and background/ cultural noise (Slater et al., 2000). These noise sources can never be eliminated entirely but there are useful techniques available for minimizing and/or quantifying the data noise. Stacking repeated measurements is useful for minimizing random errors. Reciprocal measurements (swapping the source and receiver electrode pair) can provide a measure of data precision (LaBrecque et al., 1996).

Stacking and reciprocal measurements are both useful in estimating standard deviations and/or culling outliers from the data. Understanding the noise in the data observations is crucial for data inversion, and ultimately determines how well we can resolve the subsurface structure. If noise estimates are too low, artifacts are introduced into the inverted images, while conversely, if noise estimates are too high, image resolution is degraded (LaBrecque et al., 1996).

A crucial aspect of any time-lapse problem is the ability to image the changes when repeatability of the data between surveys is in question. LaBrecque et al. (1996) observed that permanently installed electrodes are more electrically noisy when first installed, but improve over time. The higher noise levels in the initial data sets versus later data sets leads to differences in image resolution from one survey to the next. Much effort goes into making the base and subsequent data sets compatible so that data can be subtracted or normalized, however in practice this is often difficult to achieve. Oldenborger et al. (2005) showed how positioning errors in the electrodes can manifest artifacts in the inverted images.

Thus, similar to the problems with permanent electrode installation, mispositioning of electrodes can cause difficulties when working with data differences explicitly. Daily and Owen (1991) suggest inverting a normalized data set given by the following equation

$$
d_{n}=\frac{d_{t}}{d_{0}} d_{h},
$$

where $d_{n}$ is the normalized data, $d_{0}$ is the base data, $d_{t}$ is the timelapse data, and $d_{h}$ is the data that would be observed if the subsurface were a homogeneous half space.

This normalization provides a unique way to view the data changes, however, it involves a nonlinear transformation of the data and consequently, the data noise needs to be estimated due to this transformation. Instead, we advocate more straightforward linear data operations. In the next section, we discuss several ways to invert for time-lapse changes. We show that compatibility of the data sets, while desirable, is not always necessary to image the time-lapse changes. 


\section{SYNTHETIC DATA EXAMPLE}

Using a synthetic example, we now examine how one can formulate the time-lapse inversion problem to image changes in subsurface properties. The synthetic example was constructed as follows:

- We began with field data and inverted for a model, using the inversion procedure described in the next section. This model (Figure 1a) is considered the base model for the synthetic examples. The base model produced in this manner provides a realistic synthetic that is closer to the real data example than choosing an arbitrary synthetic model which is a common practice in geophysical data inversion.

- We projected this model onto a finer mesh to forward model the data. In the example presented in this paper the cell size for the forward mesh is half of the inversion mesh.

- Using this base model, we generated synthetic data and then added random noise to the data. These data are considered the base data (Figure 2a).
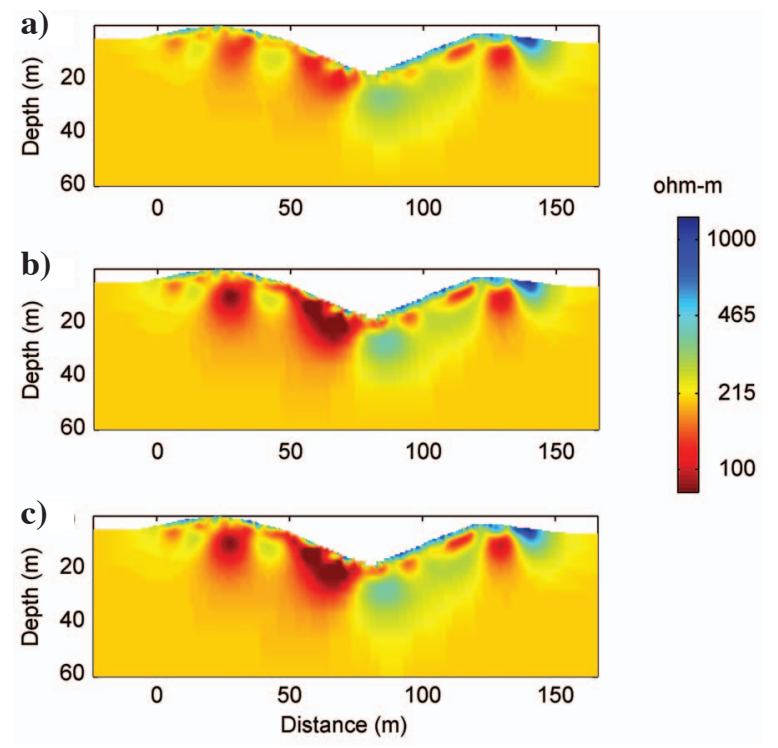

Figure 1. Synthetic example for the time-lapse inversion of resistivity data. (a) Base electrical resistivity model obtained from inversion. (b) Inverted resistivity model from time-lapse data with best fit halfspace as reference model. (c) Inverted resistivity time-lapse model with the base model in (a) as reference model.

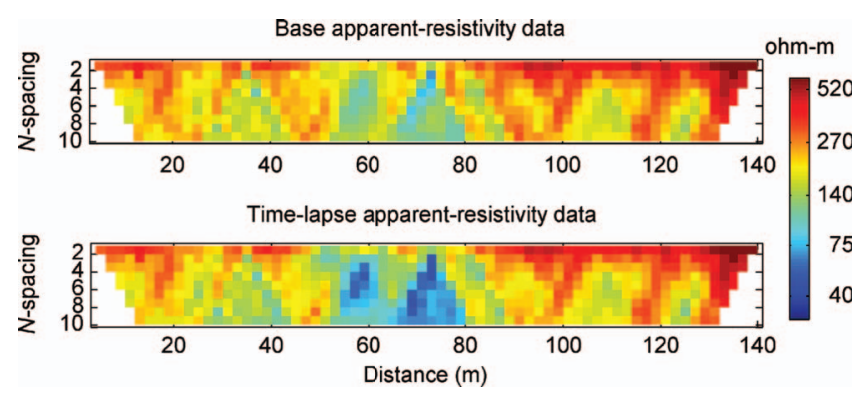

Figure 2. The base and time-lapse synthetic apparent-resistivity data contaminated with Gaussian noise with standard deviation of 5\% plus a base-level error of $1 \mathrm{mV}$.
- Next we increased the electrical conductivity by a factor of two in a localized region of the base model where changes are expected to occur to generate a time-lapse model. The regions where electrical conductivity was increased are shown in Figure 3a.

- We generated synthetic data on the finer forward mesh with the time-lapse model and then added random noise to obtain the time-lapse data (Figure 2b).

a)

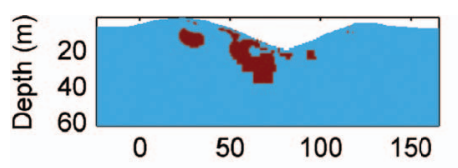

b)

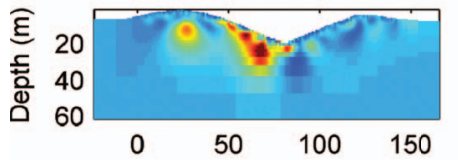

f)

c)

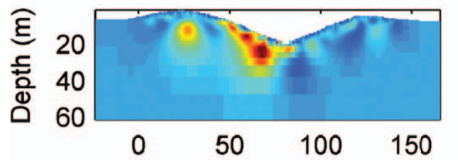

g)

d)

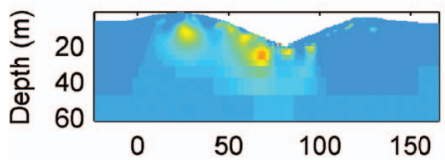

h)

e)

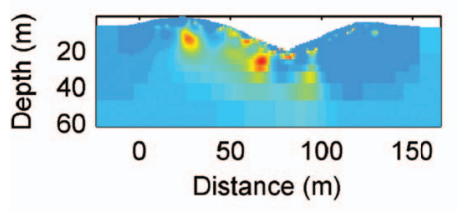

i)

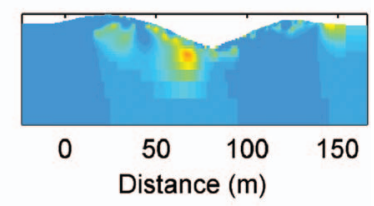

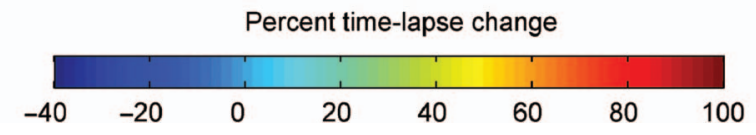

Figure 3. Synthetic example showing the region in which time-lapse change has occurred using various approaches to time-lapse inversion. (a) True model showing the changes. Left column, (b-e) base and monitor data contaminated with $5 \%$ noise. Right column, (f-i) base data has $15 \%$ noise, and monitor data has $5 \%$ noise. (b and $\mathrm{f}$ ) Time-lapse change using model subtraction. (c and g) Time-lapse change when the base model is used as the reference model for inversion of the time-lapse data. ( $\mathrm{d}$ and $\mathrm{h}$ ) Percent time-lapse change from direct inversion of the data differences (equation 6), with appropriate noise assumptions. (e and i) Percent time-lapse change from direct inversion of the data differences with lower noise assumptions so that the amplitude recovery can be enhanced. 
This approach of generating a synthetic example allows us to incorporate realistic features into the true model and yet provides us with the flexibility to examine various features of time-lapse inversion with known model changes. To investigate the different timelapse inversion approaches we consider two synthetic examples. In the first example, the base data has the same level of noise compared to the monitor data. We contaminate the base and the monitor data with Gaussian noise with a standard deviation of $5 \%$ plus a constant error floor of $1 \mathrm{mV}$.

It is possible that equipment used to acquire data between two surveys are from different vintages. For example, newer equipment typically has better signal-to-noise ratio compared to older equipment. We consider such a scenario in constructing the second synthetic example. In the second example, we contaminate the base data with Gaussian noise with standard deviation of $15 \%$ and the monitor data with $5 \%$. The base level error of $1 \mathrm{mV}$ is same for both data sets.

\section{Base model}

In the base inversion, we advocate using a simple half-space conductivity model, e.g. the best-fit half-space model, as the reference model, although this is not a strict requirement. If detailed subsurface information is available, incorporating this information into the reference model may speed convergence and improve the final images. Minimizing the objective function in equation 3 provides us with the base model denoted by $m^{\text {base }}$ shown in Figure 1a. As noted earlier, the time-lapse model is generated by increasing the conductivity by a factor of two in localized region. This is a conservative perturbation given that the conductivity of granite ranges from 1.3 $\times 10^{6} \Omega \mathrm{m}$ when dry to $4.5 \times 10^{3} \Omega \mathrm{m}$ when wet and this range of variability is observed in a variety of earth materials (Telford et al., 1990).

To track the changes to the base model we plot the quantity given by

$$
C=\left(\frac{m^{\text {base }}+\delta m}{m^{\text {base }}}\right)-1,
$$

shown in Figure 3a. The percent change in the model is essentially $C$, which can be either positive or negative. Depending on the strength of the expected time-lapse change, $C$ can be bounded. When there is no time-lapse change, $C=0$. For the synthetic example presented in this paper, $C$ is bounded between $0 \leq C \leq 100$.

\section{Model subtraction after separate inversion}

First we consider the example with the same level of noise between the base and the monitor data. The results are shown in the left panel in Figure 3. The two separate inversions were carried out on an identical finite difference mesh. The best-fit half-space conductivity model from the base inversion was utilized as the reference model in both inversions. Choosing the same reference model for both of the inversions provides a clear indication of the region where the timelapse change has occurred (see the model difference plotted in Figure $3 b$ ).

The results from the second example (higher noise level in the base data) are shown in the right panel in Figure 3. For the second example, we inverted the base and monitor data with the same reference model as in the first example. The resulting time-lapse change is shown in Figure $3 f$. Comparing Figure $3 b$ and $f$, we note the recovered time-lapse changes are very similar, however the model in Fig- ure $3 \mathrm{f}$ has more negative anomalies compared to Figure $3 \mathrm{~b}$. This is a consequence of increased noise level in the base data in the second example. As long as the noise assumptions are chosen appropriately for the respective data sets, model differencing should provide stable results.

We note that in practical time-lapse problems the changes we track are small enough that the resolution of the two data sets is nearly identical from a model resolution point of view. However, if two surveys have different resolution, then artifacts may result by taking the model differences after inversion (not shown). In the examples presented here, the base and the monitor data are inverted with the same mesh. If the parameterization of the base and monitor data are different, then model subtraction can degrade time-lapse response due to effects of regularization in the recovered models.

\section{Base model as the reference model}

As an alternative to using the same half-space reference model for each inversion, we elected to invert the base data using the best-fit half-space as the reference model, then invert the time-lapse data using the base model as the reference model (Anno and Routh, 2007; Oldenborger et al., 2007a). We refer to this method as a cascaded time-lapse inversion approach. This should effectively localize the model differences within the region that is supported by the data because all other regions will revert to the base model.

Employing this technique, we see that the regions where timelapse changes have occurred are clearly illuminated. Figure 1c shows a much closer correspondence to Figure 1a than does Figure $1 b$. We note that in both the approaches; i.e. model subtraction after separate inversions and inverting the time-lapse data with the base model as the reference; data repeatability is not a strict requirement.

It should be stated here, however, that differences in noise levels between data sets could result in different regularization of the two inversions thus one result may be much smoother than the other. In this case, the best approach would be to use the less noisy data set as the base data set, and then use approximate noise assumptions in the inversion of the time-lapse data set, although we emphasize that both inversions should converge to the same rms misfit. The cascaded approach also provides the ability to build an updated model that has the background information from the less noisy data and builds the changes on top of it. Thus the final model preserves the features obtained with the less noisy data.

Figure $3 \mathrm{c}$ and $\mathrm{g}$ shows the time-lapse change obtained using the cascaded method for the first and the second example respectively. We clearly see the time-lapse change in Figure $3 \mathrm{c}$ and $\mathrm{g}$ agree well with the true anomaly in Figure 3a. However, Figure $3 \mathrm{~g}$ has more negative anomaly artifacts due to the increased level of noise in the base data. Except very minor differences, the time-lapse results from the cascaded approach are very similar to the model subtraction approach in Figure $3 b$ and $f$.

It is important to note that the cascaded approach is more practical compared to the model subtraction approach. We expect the convergence to be faster for large 3D problems starting with the base model as reference to invert the monitor data. In the model subtraction approach, starting with a homogeneous reference model such as the best-fit half-space can be computationally intensive for large 3D problems. 


\section{Inversion of data differences}

A commonly used method in time-lapse inversion is a data difference inversion approach expressed either explicitly in terms of data differences or as a ratio (Slater et al., 2000; LaBrecque and Yang, 2001; Johnson et al., 2005, 2007). Here we difference the base data and the time-lapse data and directly invert for changes in the model. Representing the nonlinear mapping between the data and the model by $d=F(m)$ and assuming small changes in the model, a linear approximation can be used to represent the mapping between the model changes and the data changes.

We can write mathematically

$$
\begin{gathered}
\delta d=\frac{\left(F\left(m^{\text {base }}+\delta m\right)-F\left(m^{\text {base }}\right)\right)}{F\left(m^{\text {base }}\right)}=\sum_{j=1}^{M} J_{i j} \delta m_{j} \\
\text { where, } J_{i j}=\frac{1}{F\left(m^{\text {base }}\right)_{i}} \frac{\partial F_{i}\left(m^{\text {base }}\right)}{\partial m_{j}^{\text {base }}} .
\end{gathered}
$$

Equation 6 is a linearized equation, similar to the linearized IP forward model in Oldenburg and $\mathrm{Li}$ (1994). This implies that one can use a linearized IP inversion code to invert difference data in a timelapse experiment to obtain conductivity changes. Since the data changes are typically small we multiply the difference data from equation 6 by 100 and write it as percent time-lapse change.

We generate the data difference for the two synthetic examples and invert the difference data using an IP inversion code. With difference data it is not straightforward to determine what standard deviation to assign as the noise estimate. In the first example, the base and monitor data have a noise level of 5\%, therefore we choose the standard deviation for the difference data as $5 \%$ of the difference data

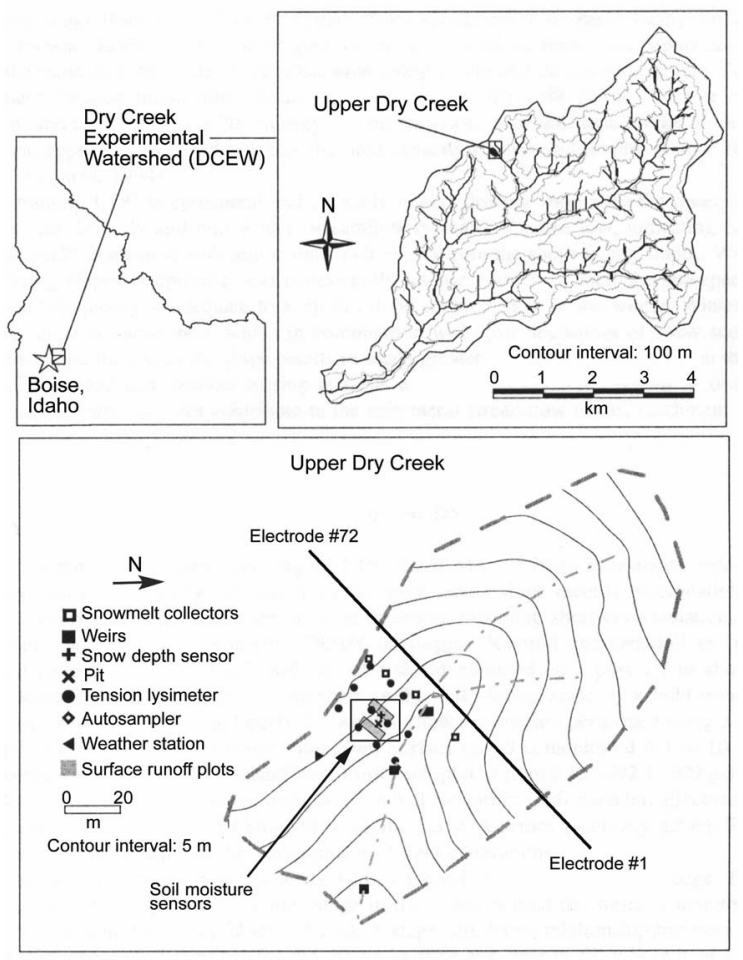

Figure 4. Location of hydrological instruments and ERT line (modified from McNamara et al., 2005). magnitude plus a base level error of $1 \mathrm{mV}$. With this noise assignment, the recovered time-lapse response from difference data is shown in Figure 3d. The inverted response is represented in terms of percent time-lapse change.

The results clearly indicate that the recovered time-lapse anomalies are lower in amplitude compared to previous approaches in Figure $3 b$ and $c$. Since the anomalies from difference inversion are lower in magnitude, we reinvert the difference data using a lower noise assumption with the hope of improving the time-lapse model. The recovered time-lapse anomalies in Figure $3 e$ show that there is an increase in amplitude of the time-lapse anomalies but it also enhances the artifacts. This is a consequence of the nature of the difference data. As a general observation, this procedure is prone to data repeatability issues because the two data are subtracted explicitly in equation 6 before inverting. Moreover, subtracting two data sets with different levels of noise can accentuate the noise for the difference data.

In the second example, the noise assignment for the difference data is somewhat questionable because the base data has more noise compared to the monitor data. As a general observation we will expect the difference data of the two data sets, i.e., base and the monitor to have noise characteristics that are higher. Based on this we choose $15 \%$ of the difference data magnitude plus a base level error of $1 \mathrm{mV}$ as the standard deviation of the data errors. The time-lapse model from the difference inversion in Figure $3 \mathrm{~h}$ clearly indicates the effect of the increased level of noise.

Comparing Figure $3 \mathrm{~h}$ with $3 \mathrm{~g}$ shows the time-lapse anomalies are reduced and the artifacts are enhanced in the difference inversion. Reinverting the same difference data with lower noise assumption produces more artifacts, shown in Figure 3i. Both synthetic examples show that the difference data inversion produces reduced anomalies and more artifacts. Thus our preferred method of time-lapse inversion is either model subtraction or inverting the time-lapse data by including the base model as the reference model during inversion.

\section{FIELD DATA EXAMPLE}

Long-term hydrological studies are being performed in a small catchment, the Treeline Site (McNamara et al., 2005), within the larger Dry Creek Experimental Watershed near Boise, Idaho, USA (Figure 4). Despite the large quantity and variety of hydrological data collected at this site, there is still only a general understanding of the groundwater flow regime within the catchment. Because of the high cost associated with installing monitoring wells and the fact that shallow bedrock prohibits piezometer installation beyond approximately one-meter depth, surface ERT is an attractive alternative method for understanding the subsurface site hydrogeology.

An ongoing question has focused on quantifying the infiltration rate of groundwater recharge, as a source and/or sink, through fracture systems within the Idaho Batholith. The goal of this study is to delineate fracture zones within the bedrock to aid in estimating infiltration rates through the fractures and, ultimately, resolve the unknown source found by hydrological models. Four ERT surveys were collected at the same location during the months of October and December 2005, April 2006, and September 2007, with the hope of imaging variations in pore water saturation within the fractures throughout the fall to summer time periods thus refining hydrologic models of the catchment.

The repeatability of the data acquired during two dry conditions (October 2005 and September 2007) and two wet conditions (December 2005 and April 2006) provide for an investigation of the 
time-lapse change of the electrical conductivity and its relation to hydrologic conditions. However, we note that the repeatability of the data sets acquired during wet conditions is better than those acquired during dry conditions and this is likely due to higher contact resistance during the dry season.

\section{Site description}

The Treeline Site encompasses $0.02 \mathrm{~km}^{2}$ and is located at a mean elevation of $1620 \mathrm{~m}$ within the Dry Creek Experimental Watershed (Figure 4). Total relief of the site is 70 meters. The Treeline Site trends northwest to southeast, and encompasses land surface slopes of $20^{\circ}-40^{\circ}$ over mostly concave and convex angles. This paper is primarily a fractured rock study, but most of the hard data that are available for the site come from a thin soil layer covering the majority of the site. Soils are derived from weathering of the Idaho Batholith (a biotite granodiorite intrusion) and are classified as sandy loam (Yenko, 2003) with depth ranges between $0.25 \mathrm{~m}$ and $1.2 \mathrm{~m}$ with an average depth of $0.45 \mathrm{~m}$. Soils are shallowest and coarsest at ridge locations along the north and northeast boundaries.

Soil depth is greatest along lower slopes adjacent to the stream channel in the center of the catchment. Texture analysis (hydrometer method) of the A, B, and $\mathrm{C}$ horizons of a 70-cm deep soil pit located midslope on a north facing aspect exhibited minimal variation in sand $(74 \%-80 \%)$, silt (15\%-17\%), and clay (7\%-9\%) (McNamara et al., 2005). The primary vegetation includes sagebrush, forbs, grasses, and scattered trees with live canopy cover ranging from $9 \%-11 \%$ during fall and winter months and $35 \%-45 \%$ in spring and summer seasons (Williams, 2005).

Precipitation (annual average of $57 \mathrm{~cm}$ ) falls mostly during the cold season, with approximately half the annual precipitation falling as snow. Rain-on-snow events are common during the late fall and early spring seasons. During typical years, persistent snow pack remains from mid-December through March with winter air temperatures ranging between $-10^{\circ} \mathrm{C}$ and $-5^{\circ} \mathrm{C}$. Summer months are hot and dry (air temperature ranges $20^{\circ} \mathrm{C}-28^{\circ} \mathrm{C}$ ) with infrequent thunderstorms.

The site drains by an ephemeral stream. Stream flow typically begins in early fall with the onset of rain, but remains low or episodic with snow pack development. Late fall and early winter rain on snow events and/or complete melt on southerly aspects generate small hydrograph peaks. Snow pack on the north-facing slope is usually maintained from the onset of snowfall. The annual hydrograph peak usually occurs in March or April depending on the duration of snowmelt (Williams, 2005). Nearby wells (within the DCEW) have been drilled to depths exceeding $100 \mathrm{~m}$ before reaching an adequately producing groundwater aquifer. Thus we do not expect to image the water table in this near-surface study.

\section{ERT data acquisition}

The ERT data were acquired using the IRIS system described previously in the Methods section. Each of the four data collection days consisted of laying out the electrical survey line along the same profile location running perpendicular to the stream axis (Figure 4). The total spread length is $144 \mathrm{~m}$ with the instrument located at the center of the spread. Spread length on each side of the instrument is $72 \mathrm{~m}$ with electrode spacing of $2 \mathrm{~m}$ for a total of 36 takeouts.

We acquired 645 quadripole measurements along the profile, using a dipole-dipole configuration with 2-m $a$-spacing and $\mathrm{N}$-spacings from 1 to 10 to take advantage of the 10-channel capabil- ity of the instrument. A minimum of four and a maximum of 16 measurements were stacked for each quadripole with a goal of $3 \%$ or lower standard deviation for the repeated measurements. We transmitted a square wave with a period of $500 \mathrm{~ms}$ and requested a potential voltage of $800 \mathrm{mV}$ on the measurement channel nearest the transmitting dipole. Data acquisition took approximately 10 minutes once the equipment was in place and the necessary parameters were input into the instrument.

Local elevation measurements were recorded at each slope break along the survey line to determine topographical variation along the survey line. Within rugged terrain, the currents injected into the ground tend to disperse beneath topographic highs and converge within topographic lows. The equipotential lines are distorted by the topography and produce false anomalies (Telford et al., 1990). By measuring the topography, we were able to account for it in the modeling and inversion, thus eliminating spurious structure in the models.

Figure 5 shows the ERT data from four different months. The repeatability of data during the wet season is better than during the dry season, but visual inspection shows that the repeatability is good in both dry and wet seasons given all the practical issues of data acquisition. Due to lower conductivities during the dry periods, some of the observed data points from October and September contained high errors (outliers) and were subsequently removed in order to minimize voltage error.

Of the 645 data values collected for each profile, 570 and 594 data values were inverted for the October and September profiles, respectively. We opted to remove the outliers from the data set because we are using the $\mathrm{L} 2$ norm as the data misfit criteria in equation 2 (alternatively, we could have assigned higher standard deviations to the outliers). All of the data values collected in December and April exhibited good data repeatability $(<6 \%$ standard deviation) because of wetter, more conductive subsurface conditions

One difficulty that we faced in this time-lapse study was that we were unable to leave the electrodes in place for the duration of the ex-

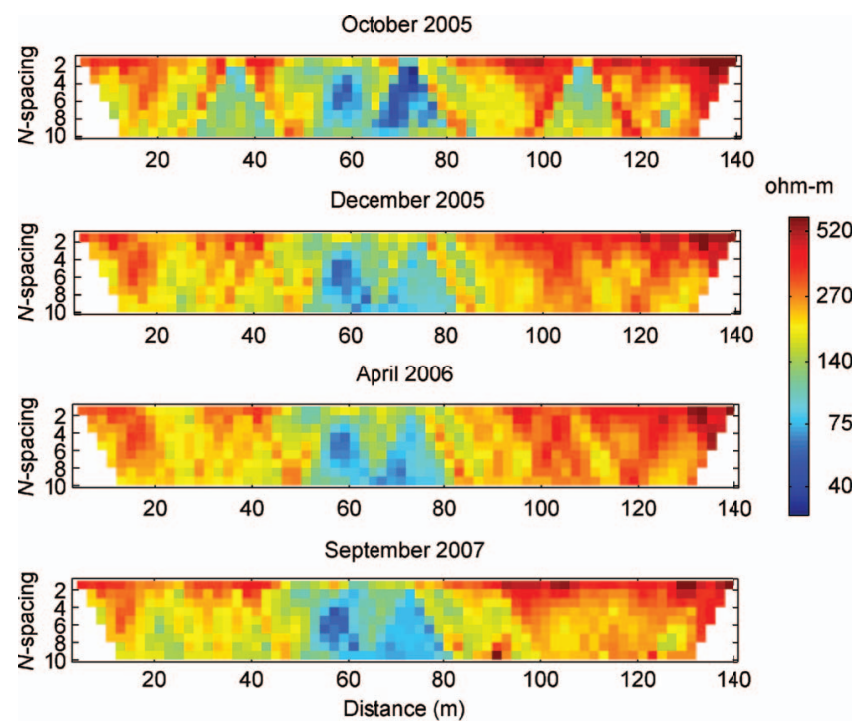

Figure 5. Apparent-resistivity data along the four ERT profiles. Note the repeatability between all four surveys, especially between the two profiles acquired in wetter conditions (December 2005 and April 2006) and the two profiles acquired in drier conditions (October 2005 and September 2007). 
periment. This resulted in slightly different electrode locations for each of the surveys. These location errors are assumed to be minor in comparison with the large differences in conductivity between the dry season and the wet season. Even if the electrode locations were permanent, the contact resistances would vary because of dramatically changing near-surface moisture conditions. This practical issue reinforces our preferred method to invert the time-lapse data, which is to use the inverted model from a base data set as the reference model for subsequent time periods.

\section{Time-lapse inversion of the Dry Creek data}

The ERT profiles were first inverted individually. Noise assumptions for the inversion were $5 \%$ of the observed data amplitudes plus a base level of $0.01 \mathrm{mV}$. Inversion was carried out as described in the methods section. Individual inversion of each data set is shown in Figure 6.

Each of the individually inverted models indicate one of three distinct conductive anomalies. The two smaller anomalies are located beneath the south and north ridgelines (centered at $23 \mathrm{~m}$ and $132 \mathrm{~m}$, respectively) and the larger, more prominent anomaly is located near the bottom of the north facing slope (centered at $65 \mathrm{~m}$; Figure 6). The inverted models show obvious similarities and differences between the dry and wet season. Profiles collected under dry conditions illustrate more distinct conductive and resistive regions, whereas the wet periods display a smoother gradient between the conductive and resistive regions (Figure 6). Persistence of the conductive anomalies in all four seasonal profiles implies that these represent actual subsurface structure rather than random artifacts.
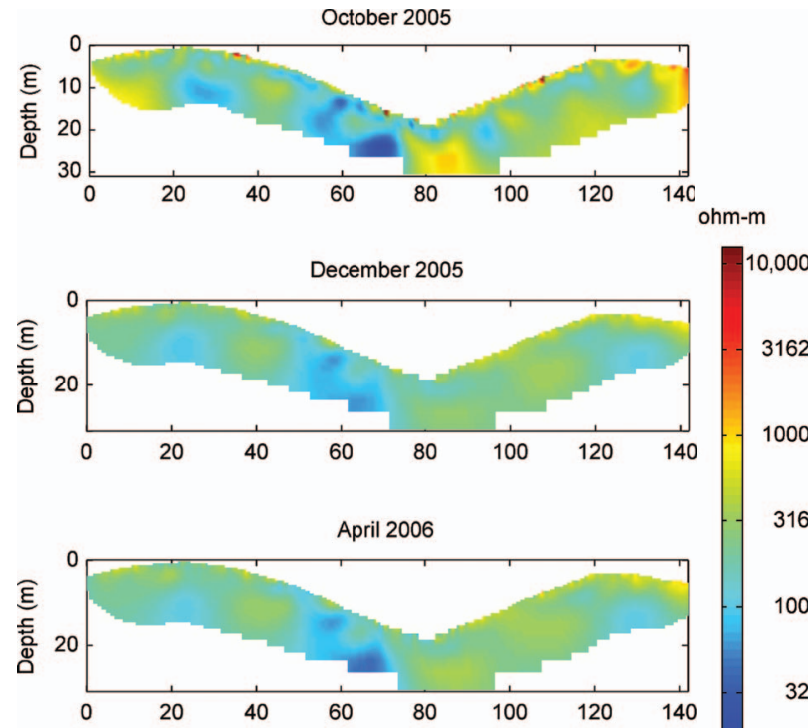

316

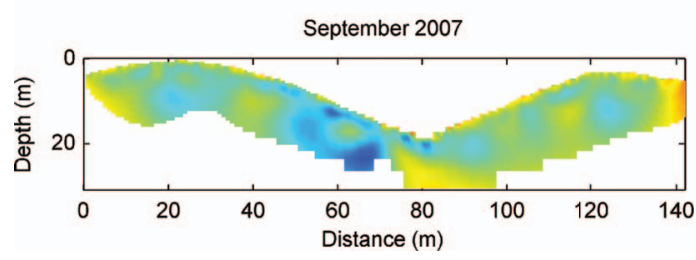

Figure 6. Inverted resisitivity models. Cool colors indicate conductive regions and warm colors indicate resistive regions. Depth of investigation for these models was determined using the DOI approach of Oldenburg and Li (1999).

\section{INTERPRETATION OF FIELD DATA RESULTS}

The volumetric moisture content is a measure of the relative amount of water in a volume of soil so that its maximum potential value is the porosity of the soil. Volumetric moisture content was measured hourly using a Campbell Scientific CR10X data logger and CS615 soil moisture sensors at depths of 5, 15, 30, 45, 60, 65, and $100 \mathrm{~cm}$. The CS615 sensors were calibrated in situ with colocated time-domain reflectometry wave guides (Chandler et al., 2004).

The soil is very dry during the summer months when the moisture content remains relatively stable near 0.07 . The 22 October 2005 survey occurred near the end of this stable dry period, prior to the onset of fall rains. Through the winter months the moisture content oscillates around 0.2. The 22 December 2005 and 14 April 2006 surveys occurred at the beginning and end of this period, after which the moisture content drops towards its summer low (Figure 7). Based on approximately two years of soil moisture data for the site, the October 2005, December 2005, and April 2006 surveys were representative samples of a prolonged dry period, a rapid wetting period, and a prolonged wet period respectively.

The persistent conductive regions within the inverted models are likely due to pore fluids in fracture zones. These anomalies are more localized in the profiles collected during dry conditions compared to the profiles gathered during water-saturated conditions. The increased conductivities within the October and September profiles could indicate presence of clays. The conductive anomalies in December and April are attributed to increased water input into the geohydrological system through precipitation that percolates through the soil column and into the fracture zones. A persistently conductive fracture zone on the south-facing slope may represent a key pathway of bedrock infiltration.

A fracture trace analysis of the Idaho Batholith conducted by Gates (1994) found three major fracture sets striking $\mathrm{N} 20^{\circ} \mathrm{E}$, $\mathrm{N} 20^{\circ} \mathrm{W}$, and $\mathrm{N} 70^{\circ} \mathrm{W}$. A more recent fracture analysis concluded a mean strike direction of joints at $\mathrm{N} 15^{\circ} \mathrm{W}$ (Hoffman et al., 2005). The $\mathrm{N} 20^{\circ} \mathrm{E}$ fracture set strikes along a similar direction to the transverse profile while the $\mathrm{N} 20^{\circ} \mathrm{W}$ and $\mathrm{N} 15^{\circ} \mathrm{W}$ fracture sets are nearly perpendicular to the profile line. The large anomaly centered at $65 \mathrm{~m}$ (Figure 6) extends approximately $20 \mathrm{~m}$ laterally and may be representative of two intersecting fracture sets $\left(\mathrm{N} 20^{\circ} \mathrm{E}\right.$ and $\left.\mathrm{N} 20^{\circ} \mathrm{W}\right)$. The remaining anomalies may be the result of intersecting fracture joints with strike between $\mathrm{N} 15^{\circ} \mathrm{W}$ to $\mathrm{N} 20^{\circ} \mathrm{W}$. This interpretation is in general agreement with what is known about the fracture system in the area (Gates, 1994; Hoffman et al., 2005).

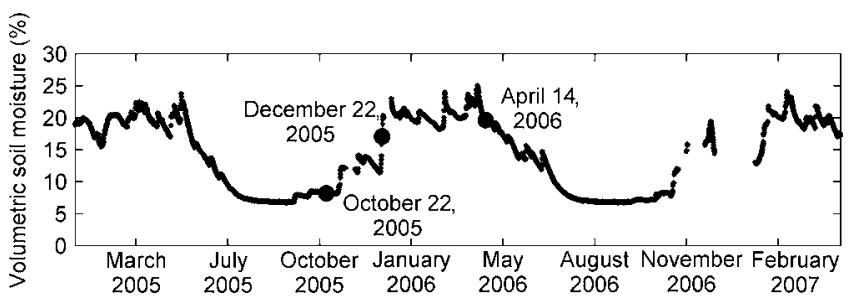

Figure 7. Volumetric soil moisture data from the study site. Data collection dates are indicated on the plot. This plot shows the average soil moisture in the approximately one-meter-thick soil column (soil moisture sensors at 5, 15, 30, 45, 60, 65, and $100 \mathrm{~cm}$ depth). 


\section{Relating the conductivity increase to an increase in water saturation}

Using a petrophysical transformation, we can relate the electrical conductivity to water saturation (see Appendix A for additional details). Because the data were collected throughout the year, this allows us to quantify the seasonal variability in water saturation at the site. Using a form of Archie's law (Archie, 1942), we rearrange the equation to solve for the water saturation $\theta$,

$$
\theta=\left(\frac{\sigma_{t}}{a \sigma_{w} \phi^{m}}\right)^{\frac{1}{n}},
$$

where $\sigma_{t}$ is the modeled conductivity; $\sigma_{w}$ is the fluid conductivity (we use $0.014 \mathrm{~S} / \mathrm{m}$ based on nearby well measurements); $\phi$ is the porosity; and $a, m$, and $n$ are fitting parameters. We used values from Carmichael (1989) for igneous rock with $4 \%$ porosity for these fitting parameters $(a=1.4, m=1.58$, and $n=2)$.

The water saturations computed for each time period are shown in Figure 8 . For the dry months, the saturation is very low except in a few compact regions. A possible explanation for these apparently highly saturated regions is that Archie's law does not work well in the presence of clays. There may be significant clay alteration of the bedrock in these areas; or alternatively, these may represent electrically conductive mineralized fracture zones. In the wet periods, the profiles appear to have a higher saturation throughout.

To better visualize the changing saturation, it is useful to look at where and how the conductivity is changing throughout the year. Figure 9 shows the percent change in conductivity from October to
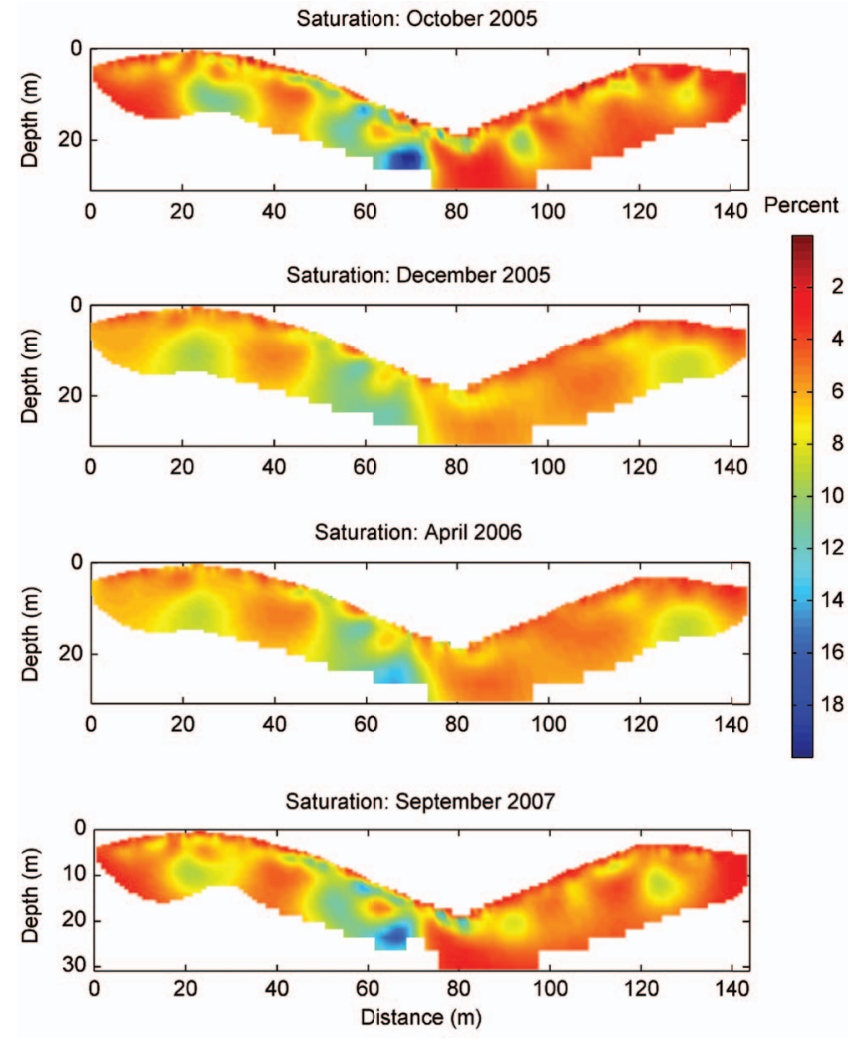

Figure 8. Water saturation in percent, calculated for each of the four conductivity models using Archie's law (equation 7). Cool colors indicate higher saturation and warm colors indicate lower saturation.
December, and from October to April. In some localized regions the conductivity decreases but globally there is an overall increase in conductivity going from the dry period in October to the wetter periods in December and April. This could be explained by transitioning from conduction paths dominated by clay content to conduction paths dominated by pore fluid saturation.

The conductivity changes of Figure 9 were used along with Equation 7 to compute the saturation increase in the model (Figure 10) from the dry season into the wet season. Note that in this case, the petrophysical relation is more meaningful because the model changes are not affected by the presence of clays. In December (Figure 10), the saturation increase is limited to a few distinct regions of the model. These regions are most likely the well-connected fracture zones, and thus quickly become more saturated when there is an increase in soil moisture. Later in the wet season, the regions of increased saturation are more pervasive through the model (Figure 10), likely due to groundwater infiltration into smaller fracture networks throughout the subsurface.

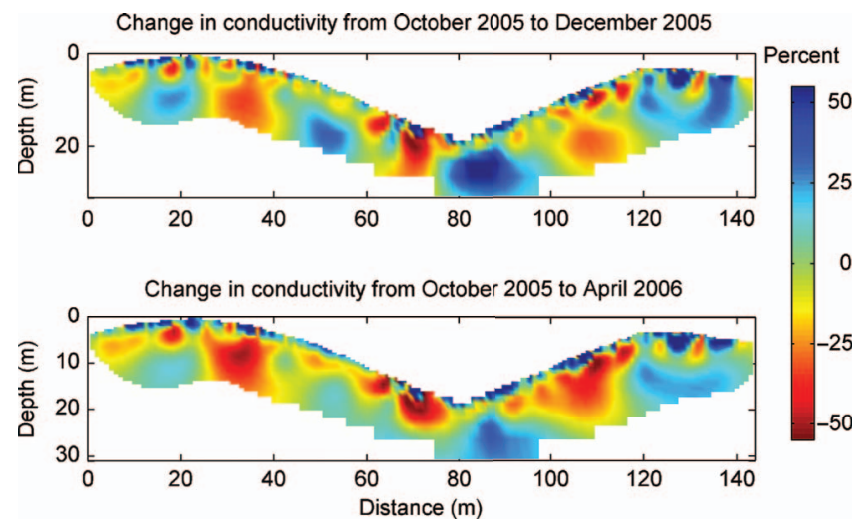

Figure 9. Percent change in conductivity from the dry season to the wet season computed using equation 5 . Cool colors indicate an increase in conductivity and warm colors indicate a decrease in conductivity.

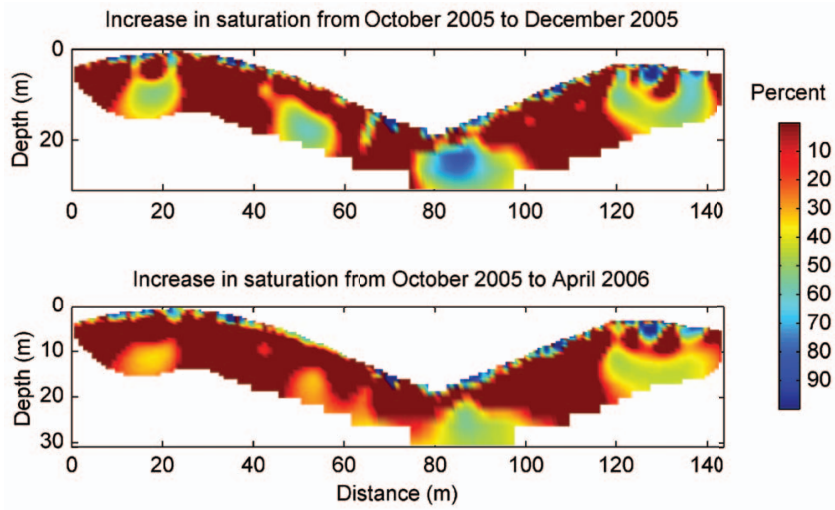

Figure 10. Percent increase in water saturation going from the dry season to the wet season. Cool colors indicate large increases in saturation and warm colors indicate small increases in saturation. Note that the largest increases in saturation are confined to the near-surface soil layer and four distinct zones within the bedrock. These four zones likely are related to fracture zones within the bedrock. 


\section{CONCLUSIONS}

Time-lapse ERT can be a very effective tool for monitoring changes in subsurface properties in an effort to understand and quantify subsurface processes. We investigate three different approaches to inverting time-lapse ERT data. As expected, the choice of the method depends strongly upon the noise levels of the base and the monitor data, i.e., the data repeatability. In the context of a smallscale watershed study, we used synthetic and field data examples to investigate the three different time-lapse ERT inversion strategies: (a) model subtraction after inverting the base and monitor data separately, (b) inverting the base data to first obtain a base model and then using it as prior information to invert the monitor data and (c) inverting data differences to recover the time-lapse anomalies.

The examples demonstrate that, of the three approaches, the data differencing approach is most sensitive to noise in the data. If data noise is well quantified and understood, and repeatability of data is good, the data differencing approach can be applied with confidence. Otherwise, the preferred approaches are model differencing or using the base model as the reference model to invert monitor data sets. These approaches, especially the latter, demonstrate a lower sensitivity to variability in data noise and thus are less susceptible to producing noise-related time-lapse artifacts.

In the field data example presented in this paper, we used static inversion of individual data sets to identify the conductive anomalies, and used the base model as the reference model to ascertain regions of increased conductivity. The regions with conductivity increase are attributed to an increase in subsurface moisture content. This time-lapse inversion methodology enabled us to identify a bedrock infiltration pathway which had previously been inferred from hydrological studies, but was not well understood. The repeatability of the data over the multiyear study and the persistent features in the inverted models provided confidence in our interpretation of this water pathway.

\section{ACKNOWLEDGMENTS}

We thank students from the GEOPH 560 course and other colleagues who helped acquire data for this project. Additional funding for this study came from NSF EPSCOR award \#EPS0132626 to PSR, and NSF EPSCOR award \#EPS0447689 to JPM. The UBCGIF developed DCIP2D, a program library for forward modeling and inversion of DC resistivity and IP data over 2D structures, developed under the consortium research project JACI. We thank Professor. Kamini Singha, two anonymous reviewers, and associate editor Professor Mark Everett for their thorough and constructive reviews.

\section{APPENDIX A}

\section{RELATION BETWEEN WATER SATURATION AND ELECTRICAL CONDUCTIVITY}

In this section we derive a relation between the change in soil moisture content and the change in electrical conducivity. The petrophysical relation between moisture and conductivity used in this work is given by Archie's law

$$
\theta=\left(\frac{\sigma_{t}}{a \sigma_{w} \phi^{m}}\right)^{\frac{1}{n}}
$$

Consider that the soil moisture changes by an amount $\delta \theta$ that results in the change in electrical conductivity by $\delta \sigma_{t}$, given by

$$
\theta+\delta \theta=\left(\frac{\sigma_{t}+\delta \sigma_{t}}{a \sigma_{w} \phi^{m}}\right)^{\frac{1}{n}}
$$

Taking the logarithm on both sides of equation A-2 and expanding we obtain

$$
\log \left[\theta\left(1+\frac{\delta \theta}{\theta}\right)\right]=\frac{1}{n} \log \left[\left(\frac{\sigma_{t}}{a \sigma_{w} \phi^{m}}\right)\left[1+\frac{\delta \sigma_{t}}{\sigma_{t}}\right]\right]
$$

$$
\begin{aligned}
\log \theta+\log \left(1+\frac{\delta \theta}{\theta}\right)= & \frac{1}{n} \log \left(\frac{\sigma_{t}}{a \sigma_{w} \phi^{m}}\right) \\
& +\frac{1}{n} \log \left(1+\frac{\delta \sigma_{t}}{\sigma_{t}}\right) .
\end{aligned}
$$

The first term on the left-hand side of equation A-4 cancels the first term on the right-hand side. Note that for $x \ll 1, \log (1+x) \approx x$, so that for small perturbations in saturation and electrical conductivity, i.e., $\delta \theta / \theta \ll 1$ and $\delta \sigma_{t} / \sigma_{t} \ll 1$, equation A-4 reduces to

$$
\frac{\delta \theta}{\theta}=\frac{1}{n} \frac{\delta \sigma_{t}}{\sigma_{t}}
$$

Therefore, $\delta \sigma_{t} / \sigma_{t}$ will directly indicate changes in relative saturation and much of the complication of the petrophysical model is simplified. Equation A-5 indicates that $\delta \theta / \theta$ is a scaled version of $\delta \sigma_{t} / \sigma_{t}$

For a general nonlinear petrophysical relation between saturation and electrical conductivity we consider

$$
\theta=f\left(\sigma_{t}, \sigma_{w}, \phi, m, a, n\right),
$$

and the change in saturation due to perturbation of conductivity is given by

$$
\theta+\delta \theta=f\left(\sigma_{t}+\delta \sigma_{t}, \sigma_{w}, \phi, m, a, n\right) .
$$

Using Taylor's series expansion and by neglecting the higher order terms, we obtain

$$
\theta+\delta \theta=f\left(\sigma_{t}\right)+\frac{\partial f}{\partial \sigma_{t}} \delta \sigma_{t}
$$

This provides a mapping between the relative change in saturation and relative change in conductivity scaled by the sensitivity of the petrophysical relation with respect to the measured changes in conductivity. This is given by

$$
\frac{\delta \theta}{\theta}=\left(\frac{\partial \log f}{\partial \log \sigma_{t}}\right) \frac{\delta \sigma_{t}}{\sigma_{t}} .
$$

If we use Archie's law, then the sensitivity is given by the scale factor $1 / n$.

$$
\frac{\partial(\log f)}{\partial\left(\log \sigma_{t}\right)}=\frac{1}{n}
$$




\section{REFERENCES}

Anno, P., and P. Routh, 2007, A new 4d workflow for legacy seismic data: 77th Annual International Meeting, SEG, Expanded Abstracts, 2888-2892.

Archie, G. E., 1942, The electrical resistivity log as an aid in determining some reservoir characteristics: Transaction of American Institute of Mining, Metallurgical, and Petroleum Engineers, 146, 54-62.

Binley, A., P. Winship, L. J. West, M. Pokar, and R. Middleton, 2002, Seasonal variation of moisture content in unsaturated sandstone inferred from borehole radar and resistivity profiles: Journal of Hydrology, 267, 160 172

Boadu, F. K., J. Gyamfi, and E. Owusu, 2005, Determining subsurface fracture characteristics from azimuthal resistivity surveys: A case study at Nsawam, Ghana: Geophysics, 70, no. 5, B35-B42.

Carmichael, R. S., 1989, Practical handbook of physical properties of rocks and minerals: CRC Press, Inc.

Chandler, D. G., M. Seyfried, M. Murdock, and J. P. McNamara, 2004, Field calibration of water content reflectometers: Soil Science Society of America Journal, 68, 1501-1507.

Daily, W., and E. Owen, 1991, Cross-borehole resistivity tomography: Geophysics, 56, 1228-1235.

Day-Lewis, F. D., J. M. Harris, and S. M. Gorelick, 2002, Time-lapse inversion of crosswell radar data: Geophysics, 67, 1740-1752.

Day-Lewis, F. D., J. W. J. Lane Jr., J. M. Harris, and S. M. Gorelick, 2003 Time-lapse imaging of saline-tracer transport in fractured rock using difference-attenuation radar tomography: Water Resources Research, 39 1290, doi: 10.1029/2002WR001722.

Descloitres, M., L. Ruiz, M. Sekhar, A. Legchenko, J.-J. Braun, M. S. M. Kumar, and S. Subramanian, 2007, Characterization of seasonal local recharge using electrical resistivity tomography and magnetic resonance sounding: Hydrological Processes, 22, 384-394

Ewing, R. P., and A. G. Hunt, 2006, Dependence of the electrical conductivity on saturation in real porous media: Vadose Zone Journal, 5, 731-741.

Fitterman, D. V., and M. T. Stewart, 1986, Transient electromagnetic sounding for groundwater: Geophysics, 51, 995-1005.

Gates, C. B., 1994, Groundwater development in granitic terrain Bogus Basin Ski Resort, Boise, Idaho: Paleogeology, Waste Disposal, Science and Politics Proceedings 30th Symposium, 55-65.

Hautot, S., P. Tarits, F. Perrier, C. Tarits, and M. Trique, 2002, Groundwater electromagnetic imaging in complex geological and topographical regions: A case study of a tectonic boundary in the French Alps: Geophysics, 67, 1048-1060.

Henry, P., 1997, Relationship between porosity, electrical conductivity, and cation exchange capacity in Barbados wedge sediments, in T. H. Shipley, Y. Ogawa, P. Blum, and J. M. Bahr, eds., Proceedings of the Ocean Drilling Program, Scientific Results 156137-149, doi: 10.2973/ odp.proc.sr.156.020.1997

Hoffman, B., P. Antrim, J. McNamara, C. Northrup, and D. Wilkins, 2005 , Multiscale fracture analysis of the Idaho Batholith, Dry Creek experimental watershed, Geological Society of America, Abstracts with Programs, 37, no. 7, 371 .

Hubbard, S. S., K. Grote, M. Kowalsky, and Y. Rubin, 2001, Investigating temporal and spatial variations in near surface water content using GPR EOS, Transactions, American Geophysical Union 82, Abstract GP11B-09.

Hubbard, S. S., and Y. Rubin, 2006, Hydrogeological characterization using geophysical methods, in J. Delleur, ed., Handbook of groundwater engineering: CDC Press, section 14-1.

Johnson, T. C., P. S. Routh, W. Barrash, and M. D. Knoll, 2007, A field comparison of Fresnel zone and ray-based GPR attenuation-difference tomography for time-lapse imaging of electrically anomalous tracer or contaminant plumes: Geophysics, 72, no. 2, G21-G29.

Johnson, T. C., P. S. Routh, and M. D. Knoll, 2005, Fresnel volume georadar attenuation-difference tomography: Geophysical Journal International, $162,9-24$

LaBrecque, D. J., M. Miletto, W. Daily, A. Ramirez, and E. Owen, 1996, The effects of noise on Occam's inversion of resistivity tomography data: Geophysics, 61, 538-548.

LaBrecque, D. J., and X. Yang, 2001, Difference inversion of ERT data: A fast inversion method for 3-D in situ monitoring: Journal of Environmental and Engineering Geophysics, 6, 83-89.

Lane, J. W. J., F. D. Day-Lewis, and C. C. Casey, 2006, Geophysical monitoring of a field-scale biostimulation pilot project: Ground Water, 44 440-443.

Lane, J. W. J., F. P. Haeni, and W. M. Watson, 1995, Use of a square-array di- rect-current resistivity method to detect fractures in crystalline bedrock in New Hampshire: Ground Water, 33, 476-485.

Lumley, D. E., 2001, Time-lapse seismic reservoir monitoring: Geophysics, 66, 50-53.

MacBeth, C., M. Floricich, and J. Soldo, 2006, Going quantitative with 4D seismic analysis: Geophysical Prospecting, 54, 303-317.

McNamara, J. P., D. Chandler, M. Seyfried, and S. Achet, 2005, Soil moisture states, lateral flow, and streamflow generation in a semi-arid, snowmelt-driven catchment: Hydrological Processes, 19, 4023-4038.

McNeill, J. D., 1990, Use of electromagnetic methods for groundwater studies, in S. H. Ward, ed., Geotechnical and environmental geophysics, SEG, $107-112$.

Miller, C., and P. Routh, 2007, Resolution analysis of geophysical images: Comparison between point spread function and region of data influence measures: Geophysical Prospecting, 55, 835-852.

Mohnke, O., K. Prokoph, and U. Yaramanci, 2006, Electrical resistivity tomography (ERT) as a tool for monitoring moisture dynamics in soil filled containers: Proceedings of 12th European Meeting of Environmental and Engineering Geophysics, B026.

Mualem, Y., and S. P. Friedman, 1991, Theoretical prediction of electrical conductivity in saturated and unsaturated soil: Water Resources Research, 27, 2771-2777.

Müller, M., O. Mohnke, J. Schmalholz, and U. Yaramanci, 2003, Moisture assessment with small-scale geophysics — The Interurban Project: Near Surface Geophysics, 1, 171-182.

Oldenborger, G. A., M. Knoll, P. Routh, and D. LaBrecque, 2007a, Timelapse ERT monitoring of an injection/withdrawal experiment in a shallow unconfined aquifer: Geophysics, 72, no. 4, F177-F187.

Oldenborger, G. A., P. S. Routh, and M. D. Knoll, 2005, Sensitivity of electrical resistivity tomography data to electrode position errors: Geophysical Journal International, 163, 1-9.

-2007b, Model reliability for 3D electrical resistivity tomography: Application of the volume of investigation index to a time-lapse monitoring experiment: Geophysics, 72, no. 4, F167-F175.

Oldenburg, D. W., and Y. Li, 1994, Inversion of induced polarization data: Geophysics, 59, 1327-1341.

, 1999, Estimating the depth of investigation in DC resistivity and IP surveys: Geophysics, 64, 403-416.

Porsani, J. L., V. R. Elis, and Y. Hiodo, 2005, Geophysical investigations for the characterization of fractured rock aquifers in Itu, SE Brazil: Journal of Applied Geophysics, 57, 119-128.

Ramirez, A., W. Daily, D. LaBrecque, E. Owen, and D. Chesnut, 1993, Monitoring an underground steam injection process using electrical resistance tomography: Water Resources Research, 29, 73-87.

Ramirez, A. L., W. D. Daily, and R. L. Newmark, 1995, Electrical resistance tomography for steam injection monitoring and process control: Journal of Environmental and Engineering Geophysics, 0, 39-527.

Sharma, S. P., and V. C. Baranwal, 2005, Delineation of groundwater-bearing fracture zones in a hard rock area integrating very low frequency electromagnetic and resistivity data: Journal of Applied Geophysics, 57, 155166.

Singha, K., and S. M. Gorelick, 2005, Saline tracer visualized with three-dimensional electrical resistivity tomography: Field-scale spatial moment analysis: Water Resources Research, 41, W05023, doi: 10.1029/ 2004WR003460.

Slater, L. D., A. Binley, and D. Brown, 1997, Electrical imaging of fractures using ground-water salinity change: Ground Water, 35, 436-442.

Slater, L., A. M. Binley, W. Daily, and R. Johnson, 2000, Cross-hole electrical imaging of a controlled saline tracer injection: Journal of Applied Geophysics, 44, 85-102.

Telford, W. M., L. P. Geldart, and R. E. Sheriff, 1990, Applied geophysics, 2nd ed.: Cambridge University Press.

Tsourlos, P., R. Ogilvy, P. Meldrum, and G. Williams, 2003, Time-lapse monitoring in single boreholes using electrical resistivity tomography: Journal of Environmental and Engineering Geophysics, 8, 1-14.

Williams, C. J., 2005, Characterization of the spatial and temporal controls on soil moisture and streamflow generation in a semi-arid headwater catchment: M.S. thesis, Boise State University.

Yenko, M., 2003, Hydrometric and geochemical evidence of streamflow sources in the Upper Dry Creek Experimental Watershed, southwestern Idaho: M.S. thesis, Boise State University.

Zhou, Q. Y., J. Shimada, and A. Sato, 2001, Three-dimensional spatial and temporal monitoring of soil water content using electrical resistivity tomography: Water Resources Research, 37, 273-286. 